\title{
Dependencia del celular, hábitos y actitudes hacia la lectura y su relación con el rendimiento académico
}

\section{Cell phone dependence, habits and attitudes towards reading and its relationship with academic performance}

\author{
Jorge A. Hilt ${ }^{1}$ \\ Instituto Adventista Mariano Moreno, Posadas, Misiones, Argentina ${ }^{1}$ \\ Orcid ID: https://orcid.org/0000-0002-5860-2982 ${ }^{1}$
}

Recibido: 15 de marzo 2019

Aceptado: 07 de julio 2019

\begin{abstract}
Resumen
El celular (móvil) ha llegado a formar parte de la vida cotidiana de los jóvenes y su uso continúa en expansión, siendo utilizado cada vez a una menor edad. Al mismo tiempo, el uso del celular puede llegar a convertirse en un distractor cuando se lo utiliza en demasía con fines de entretenimiento, especialmente cuando compite con el tiempo que se debe destinar a la lectura y las tareas escolares. Por ello, este estudio trató de establecer si existía una relación entre el uso del celular, los hábitos y actitudes hacia la lectura y su influencia en el rendimiento académico en alumnos de nivel medio del Instituto Adventista Mariano Moreno, de la ciudad de Posadas, Misiones, en el ciclo lectivo 2018. El estudio fue de tipo transversal, correlacional y cuantitativo. Para medir el uso del celular se utilizó el Test de Dependencia al Móvil (TDM); las actitudes y hábitos hacia la lectura fueron valoradas con el cuestionario HAL, y el rendimiento académico se obtuvo por medio de la calificación del primer trimestre del ciclo lectivo 2018. La muestra estuvo constituida por 126 estudiantes de primero a quinto año, que representan el $66.67 \%$ del alumnado de nivel medio. Se encontró evidencia de que existe una relación negativa entre la dependencia del celular y las otras variables del estudio: (a) hábitos de lectura, (b) actitudes hacia de lectura y (c) rendimiento académico. En lo referente al género, el grupo de alumnas obtuvo mayores puntuaciones en todas las variables del estudio. Aunque las mujeres hacen un mayor uso del celular y tienen una percepción mayor de dependencia, eso no les impide obtener mejores calificaciones que los varones. Casi la mitad de la muestra utiliza Instagram como red social de preferencia.
\end{abstract}

Palabras clave: adicción al móvil, hábitos de lectura, actitud hacia la lectura, rendimiento académico. 


\begin{abstract}
Cell phone (mobile) has become part of the daily life of young people and its use continues to expand, being used every time at a younger age. At the same time, the use of the cell phone can become a distraction when it is used too much for entertainment purposes, especially when it competes with the time that should be spent on reading and doing homework. Therefore, this study tried to establish whether there was a relationship between cell phone use, habits and attitudes towards reading and its influence on academic performance in middle-level students of the Mariano Moreno Adventist Institute, in the city of Posadas, Misiones (Argentina), in the school year 2018. The study was cross-sectional, correlational and quantitative. To measure the use of the cell phone, the Mobile Dependency Test (TDM) was used; Attitudes and habits towards reading were valued with the HAL questionnaire, and academic performance was obtained by means of the first quarter qualification of the 2018 school year. The sample consisted of 126 students from first to fifth year, representing $66.67 \%$ of middle-level students. We found evidence that there is a negative relationship between cell phone dependence and the other study variables: (a) reading habits, (b) attitudes towards reading and (c) academic performance. Regarding gender, the group of students obtained higher scores in all the variables of the study. Although women make greater use of cell phones and have a greater perception of dependence, this does not prevent them from obtaining better grades than men. Almost half of the sample uses Instagram as the preferred social network.
\end{abstract}

Keywords: mobile addiction, reading habits, attitude towards reading, academic performance.

\title{
Introducción
}

Dependencia del celular

El celular se ha convertido en un aparato muy importante en la vida de las personas, ya que supera tanto a los televidentes como a los internautas (García Martínez y Fabila Echauri, 2014). Ha resultado ser una herramienta muy útil para estar conectados con otras personas y ha favorecido el conocimiento de los sucesos del mundo en tiempo real (Mendoza Méndez, Pérez Chávez, Jaramillo Jaramillo y Baena Castro, 2018).

No obstante, en los últimos años se han observado dificultades por el grado de adicción al celular que genera su uso indiscriminado, especialmente en los adolescentes y jóvenes. De manera especial, las mujeres y los adolescentes en general, tienen un mayor grado de dependencia del celular a partir de los 15 años. Los estudios muestran que el uso excesivo e inadecuado del celular también interfiere en el desarrollo interpersonal, que se manifiesta con la pérdida de contacto cara a cara con el entorno social (Gamero et al., 2016), lo cual es una clara afectación de la conducta, que tiene consecuencias a nivel personal, social y familiar. 
La adicción no se produce por la frecuencia del uso del celular, sino por la relación de dependencia que genera. Existen estudios que muestran una relación entre la adicción al celular y la depresión e inestabilidad emocional, el síndrome de túnel carpiano, el trastorno del sueño (Guillén Cadena, Beltrán Lugo, Aristizábal Hoyos y Garduño Magaña, 2016), el consumo de alcohol y el fracaso escolar (García Martínez y Fabila Echauri, 2014). Incluso, muchos sujetos adictos al celular tienen temor de llegar a perderse de alguna novedad de su entorno, lo cual explica en parte por qué están ansiosos por las notificaciones que llegan al móvil.

El celular se ha convertido en un instrumento muy atractivo, al incorporar cámara fotográfica, grabadora de voz, infinidad de aplicaciones y navegación por internet. La búsqueda de contenidos multimedia ha dejado de estar limitada al uso de la PC, para pasar a los dispositivos móviles como celulares, tablets, notebooks, kínder, etc. (Marcelino Bento y dos Santos Cavalcante, 2013). Además, en el celular se puede tener acceso casi a cualquier cosa: cuentas bancarias, calculadoras científicas, bibliotecas virtuales, e infinidad de páginas de uso educativo, recreativo, laboral, etc.

En el ámbito escolar, existen nuevas tendencias que tratan de incorporar el celular como herramienta de búsqueda de información. Algunos encuentran en el celular la herramienta más adecuada para compartir archivos, o lo ven como un dispositivo que contribuye al trabajo colaborativo. Pero la tecnología trae consigo un dilema, que tiene que ver el modo de uso, es decir, cuán permisivo debe ser el uso de las TICs en el aula, ya que la herramienta tiene tanto potencial para lo educativo como para otros usos no deseados (Marcelino Bento y dos Santos Cavalcante, 2013).

Un estudio realizado por Henríquez Ritchie, Organista Sandoval y Lavigne (2013), buscó estimar los procesos de interacción social y de interactividad con la información de estudiantes y docentes de la Universidad Autónoma de Baja California, México. Los estudiantes señalaron, respecto a la interacción social, que usan el celular para llegar a acuerdos, organizar tareas y aclarar dudas sobre temas escolares, mientras que los docentes lo utilizan para comunicarse con colegas o acordar actividades para los estudiantes. Respecto a la interactividad con la información, tanto estudiantes como docentes señalaron que la búsqueda y recuperación de información eran los principales usos que hacían del celular.

Una preocupación que se da en el ámbito escolar, es el traspaso del fenómeno del bullying desde el aula a las redes sociales. La agresión, el acoso y la difamación que comienzan en el aula se magnifican en las redes sociales, de tal manera que la humillación 
se incrementa junto a los observadores que lo comparten con otros amigos virtuales (Castelli Olvera y Rosa, 2018). Aunque las adicciones tecnológicas generalmente se asocian con una diversidad de variables psicológicas, González Cortijo (2012) no encontró evidencia que permita afirmar que la adicción al móvil influya en el resultado académico. En lo que se refiere al género, las mujeres parecen utilizar más el móvil y tienen una mayor dependencia que los varones.

\section{Actitud hacia la lectura}

La lectura es un proceso complejo, mediante el cual los símbolos impresos, escritos o virtuales, llegan a tener un significado para el lector, quién deberá interpretar y comprender lo que lee. No se trata de vocalizar o reconocer las palabras en oraciones o párrafos, porque esto no es garantía de comprensión de las mismas; sino de comprender su significado, interpretar, evaluar, reflexionar y aprovechar lo que se lee (Lainfiesta Stricker, 2006). Leer implica el dominio de la decodificación de las palabras y la comprensión del significado de las frases por medio de la habilidad de extraer las ideas principales del texto escrito (Pinzás, en Aliaga Jiménez, 2012).

Por otro lado, Aiken comenta (2003) que las actitudes están relacionadas con las creencias, los sentimientos y la conducta. Estas tienen su origen en los pensamientos y sentimientos, que afectan la percepción de la realidad y el comportamiento (Ponce Aguirre, 2010). En teoría, una actitud es una tendencia a reaccionar de manera positiva o negativa hacia algún objeto, persona o situación. Existen tres conceptos que están íntimamente relacionados en los modelos de comprensión de las actitudes, y son los siguientes: (a) conocimientos o creencias, (b) sentimientos y (c) conductas (Aiken, 2003; McKenna, Kear y Ellsworth, 1995). Las actitudes se originan en los pensamientos y sentimientos, que afectan la percepción que la persona tiene de la realidad y, por lo tanto, del comportamiento (Ponce Aguirre, 2010). El modelo de Mathewson (1994, citado en McKenna et al., 1995) integra estos tres elementos para comprender lo que es la actitud. Este modelo propone que la actitud hacia la lectura está influenciada por una serie de factores que afectan la intención de leer o de seguir leyendo.

En otro orden, los conocimientos, llamados también componentes cognoscitivos (Aiken, 2003), se refieren a lo que el individuo cree que es cierto; de allí que el término también se utiliza como sinónimo de creencias (Morales Vallejo, 2011). No se trata de verificar lo que el individuo conoce y cree, o si los datos son ciertos; sino si su actitud es positiva o negativa. Por eso, las conductas hacia la lectura se refieren más a la intención 
de leer, al deseo de leer, a lo que uno haría, y no a la conducta misma (Morales Vallejo, 2011).

El término actitud se refiere a la evaluación bipolar o dimensión afectiva de una persona con respecto a algún objeto, acción o evento. Representa un sentimiento general de una persona, que puede ser favorable o desfavorable respecto del objeto estímulo, que es afectado por las creencias hacia ese objeto (Fishbein y Ajzen, 1975). Alexander y Filler (citados en Bokhorst-Heng y Pereira, 2008), definen la actitud hacia la lectura como un sistema de sentimientos relacionados con la lectura, que hace que el estudiante se acerque (sentimiento positivo) o evite (sentimiento negativo) un momento de lectura.

En consecuencia, uno de los mayores retos que hay en el mundo, especialmente en países no desarrollados, es cómo llevar la lectura a personas que no leen. En la actualidad, con la presencia de dispositivos móviles, la situación del acceso a la lectura se está democratizando, aunque sólo el $40 \%$ de la población mundial tiene acceso a internet. El dilema es cómo llevar textos a los que se mantienen desconectados en zonas marginales (UNESCO, 2015).

\section{Hábitos de lectura}

El hábito de la lectura se refiere a la práctica o costumbre de leer. Es el grado en que las personas leen con regularidad, al punto de formar una costumbre o práctica frecuente de lectura. La práctica habitual de la lectura permite identificar frecuencias, intensidades, modos, lugares, etc. (Tejada, 2008). Si la práctica de leer sucede sólo cuando hay supervisión externa, no se convierte en un hábito duradero (Peiró i Gregòri et al., 2009).

La mayoría de los instrumentos sobre hábitos de lectura son cuestionarios de preguntas de opción múltiple que indagan acerca de cuánto leen los individuos, frecuencia de lectura, qué leen, si asisten a la biblioteca o si sus padres leen en casa. De esta manera, tratan de medir la frecuencia lectora para determinar si existe o no el hábito de la lectura.

\section{Rendimiento académico}

El rendimiento académico es el resultado de múltiples factores que intervienen en el alumno, y que se reflejan en el valor o calificación numérica, lo cual puede incidir en el abandono, retraso o éxito en los estudios (Garbanzo Vargas, 2007). 


\section{Análisis del problema}

Esta investigación buscó dar respuesta a la siguiente pregunta: ¿existe relación entre la dependencia del celular, los hábitos y actitudes hacia la lectura con el rendimiento académico de los estudiantes de nivel secundario del Instituto Adventista Mariano Moreno, de la ciudad de Posadas, en el ciclo lectivo 2018 ?

\section{Hipótesis}

La hipótesis de este estudio afirma que existe relación significativa entre la dependencia del celular, los hábitos y actitudes hacia la lectura con el rendimiento académico de los estudiantes de nivel secundario del Instituto Adventista Mariano Moreno, de la ciudad de Posadas, en el ciclo lectivo 2018.

\section{Objetivos}

Los objetivos de esta investigación fueron los siguientes:

- Determinar si existe relación entre la dependencia del celular y el rendimiento académico.

- Determinar si existe relación entre la dependencia del celular y los hábitos de lectura y la actitud hacia la lectura.

- Determinar si existe relación entre los hábitos de lectura, las actitudes hacia la lectura con el rendimiento académico.

- Determinar si existe diferencia en las variables entre varones y mujeres.

\section{Metodología}

La metodología se refiere al diseño que se eligió para desarrollar la investigación. En este caso, fue de tipo transversal, correlacional y cuantitativa. Fue transversal, porque se recogieron los datos en un momento específico, sin hacer un seguimiento en el tiempo. Fue correlacional, porque trató de establecer si había correlación entre las variables del estudio. Por último, fue de tipo cuantitativa, porque los datos recolectados fueron analizados con el auxilio de la estadística.

\section{Instrumento}

Para la recolección de los datos se utilizó el Cuestionario de Hábitos y Actitudes hacia la Lectura (Hilt, 2013), que consta de dos partes: 
1. Una sección de Actitudes hacia la lectura, con 30 ítems valorados en una escala de cinco opciones que va desde totalmente en desacuerdo hasta totalmente de acuerdo.

2. Una sección de Hábitos de Lectura, con 10 puntos que indagan sobre las costumbres relacionadas con la lectura.

Además, se utilizó el Test de Dependencia del Móvil (TDM), de Chóliz y Villanueva (2011), que consta de 22 ítems, valorados con una escala Likert de cinco opciones.

\section{Muestra}

La muestra fue no-probabilística y se recogieron los datos de alumnos que estuvieron presentes el día de la aplicación del instrumento, de primero a quinto año de nivel secundario del Instituto Adventista Mariano, provincia de Misiones, Argentina. Esta población de estudio estuvo compuesta por 126 estudiantes.

\section{Resultados}

Datos demográficos

En cuanto a la edad, la muestra tuvo un rango que va de 13 a 18 años, con una media de 14.82 años y una desviación estándar de 1.335 (ver Tabla 1). Los alumnos pertenecían a los cinco cursos de nivel secundario: (a) $1^{\circ}$ año, con 32 alumnos (25.4\%); (b) $2^{\circ}$ año, con 28 alumnos (22.2\%); (c) $3^{\circ}$ año, con 25 alumnos (35.7\%); (d) $4^{\circ}$ año, con 30 alumnos $(23.8 \%)$ y (e) $5^{\circ}$ año, con 11 alumnos (8.7\%). En cuanto al género, 50 alumnos fueron varones $(39.7 \%)$ y 76 fueron mujeres $(60,3 \%)$, como se puede apreciar en la Figura 1.

\section{Resultados de los instrumentos TDM}

El análisis de confiabilidad del TDM, medido por el alfa de Cronbach, alcanzó un resultado de 0.888 para los 22 ítems, que fueron medidos con una escala tipo Likert de cinco opciones. En el análisis factorial los ítems se agruparon en seis factores: 
Tabla 1

Resultados de la muestra por edad

\begin{tabular}{ccc} 
Edad & $n$ & $\%$ \\
\hline 13 & 27 & 21.4 \\
14 & 28 & 22.2 \\
15 & 27 & 21.4 \\
16 & 30 & 23.8 \\
17 & 13 & 10.3 \\
18 & 1 & .8 \\
\hline Total & 126 & 100.0
\end{tabular}

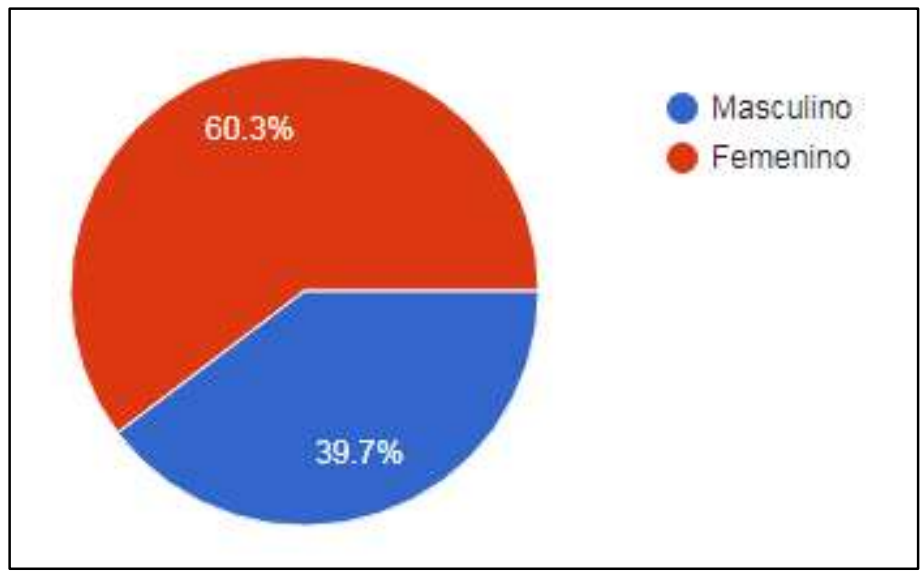

Figura 1. Distribución de la muestra por género

1. Abstinencia (6 ítems), que se manifiesta por un severo malestar que se provoca cuando no se tiene la posibilidad de utilizar el móvil o hace tiempo que no se puede usar.

2. Tolerancia ( 3 ítems), se refiere a la necesidad de utilizar cada vez más el celular para conseguir la misma satisfacción que al principio o que el uso moderado no llegue a ser suficiente para la persona.

3. Impulsividad (4 ítems), se refiere la dificultad en controlar el impulso, es decir, a la utilización excesiva del móvil en cualquier momento y situación.

4. Abuso y dificultad en el uso (4 ítems), se refiere al uso excesivo que tiene mucho que ver con la dificultad de controlar la conducta. Las nuevas aplicaciones del móvil, especialmente las de mensajería, dificultan enormemente el control conductual.

5. Problemas familiares ( 3 ítems), relacionado con el costo tanto del aparato como del crédito necesario para el funcionamiento del celular. 
6. Problemas por el tiempo de uso ( 2 ítems), indaga sobre el tiempo que se usa el celular, que puede incluso a hacer perder horas de sueño.

Resultados de la prueba HAL

El cuestionario HAL mide los hábitos y actitudes hacia la lectura (Hilt, 2013). El análisis de confiabilidad del HAL, medido por el alfa de Cronbach, obtuvo un resultado de 0.94 para la sección de actitudes hacia la lectura. Esta sección fue medida mediante una escala tipo Likert de cinco opciones, que quedó formada por 29 ítems agrupados en tres dimensiones:

1. Sentimientos hacia la lectura (9 ítems), referidos al gusto y el placer que produce leer.

2. Creencias acerca de la lectura (8 ítems), que expresan lo que la persona cree que produce la lectura en él o ella.

3. Conductas relacionadas con la lectura (12 ítems), menciona lo que la persona hace en relación a la lectura.

La sección de hábitos de lectura estuvo formada por 9 apartados, cuyo puntaje máximo sumó 33 puntos, y que trató sobre los siguientes ítems:

1. Cantidad de horas dedicadas a la lectura escolar.

2. Cantidad de horas semanales dedicadas a la lectura no escolar.

3. Hábito de leer en el tiempo libre.

4. Cantidad de libros leídos en el último año.

5. Mayor o menor hábito de lectura comparado con el año anterior.

6. Completa o no los libros que se empiezan a leer.

7. Medios físicos preferidos para la lectura.

8. Tipo de libros que lee con mayor frecuencia.

9. Te leían historias cuando eras chico.

\section{Prueba de hipótesis}

La hipótesis de nuestra investigación declara que existe relación entre la adicción al celular, los hábitos y actitudes hacia la lectura con el rendimiento académico de los estudiantes de nivel secundario del Instituto Adventista Mariano Moreno, de la ciudad de Posadas, en el ciclo lectivo 2018. 
A fin de poner a prueba la hipótesis, se procedió al análisis de correlaciones, para determinar si la adicción al celular, los hábitos y actitudes hacia la lectura se relacionaban con el promedio académico de los estudiantes que participaron de este estudio. Los resultados mostraron que la adicción al celular se relaciona significativamente con:

(a) Los hábitos de lectura ( $r=-.184, p=.039)$, es decir que, a mayor adicción al celular, menores son los hábitos de lectura.

(b) La actitud hacia la lectura $(r=-.230, p=.009)$, esto significa que, a mayor adicción al celular, menor es la actitud hacia la lectura.

(c) El promedio académico $(r=-.207, p=.020)$, esto quiere decir que, a mayor adicción al celular, menor es el promedio académico. Todas las relaciones fueron negativas o inversas, es decir, que a mayor valoración de una variable (adicción al celular), menores eran las valoraciones de las otras variables (ver Tabla 2).

Tabla 2

Correlación entre la adicción al celular (TDM), los hábitos de lectura y las actitudes hacia la lectura con el rendimiento académico (Promedio)

\begin{tabular}{llrrrr}
\hline \multicolumn{5}{c}{ Correlaciones } \\
\hline \multirow{2}{*}{ Hab_Lect } & r de Pearson & Hab_Lect & TDM & Act_Lect & Promedio \\
& $P$ & 1 & $-.184^{*}$ & $.536^{* *}$ & .037 \\
TDM & r de Pearson & $-.184^{*}$ & .039 & .000 & .682 \\
& $\mathrm{P}$ & .039 & 1 & $-.230^{* *}$ & $-.207^{*}$ \\
Act_Lect & $\mathrm{r}$ de Pearson & $.536^{* *}$ & $-.230^{* *}$ & .009 & .020 \\
& $\mathrm{P}$ & .000 & .009 & 1 & $.213^{*}$ \\
Promedio & $\mathrm{r}$ de Pearson & .037 & $-.207^{*}$ & $.213^{*}$ & .017 \\
& $\mathrm{P}$ & .682 & .020 & .017 & 1 \\
\hline
\end{tabular}

Uno de los objetivos propuestos era determinar si había diferencia entre varones y mujeres en las variables del estudio. Los resultados mostraron que existe una diferencia significativa en la dependencia del móvil $\left(t_{(112.886)}=-2.556, p=.012\right)$ entre varones $(M=$ 2.54) y mujeres $(M=2.81)$. Además, se observó una diferencia significativa en el promedio académico $\left(t_{(111.271)}=-3.711, p=.000\right)$ del primer trimestre de 2018 entre varones $(M=7.34)$ y mujeres $(M=8.01)$ que participaron del estudio. Otro resultado señala una diferencia significativa en la actitud hacia la lectura $\left(t_{(107.071)}=-3.093, p=\right.$ 
$.003)$ entre varones $(M=3.34)$ y mujeres $(M=3.72)$; sin embargo, no se encontró una diferencia significativa en la media de los hábitos de lectura entre varones y mujeres.

\section{Otros resultados}

El cuestionario online incluyó una serie de preguntas relacionadas con el uso del celular. A continuación, se exponen las respuestas a esos interrogantes:

1. Se consultó cuál es la aplicación que los alumnos utilizaban con mayor frecuencia. Las aplicaciones con mayor frecuencia de uso fueron: (a) Instagram (47.6\%), (b) WhatsApp (29.4\%), (c) Youtube (17.5\%).

2. Otra pregunta consistió en saber si los alumnos tenían paquete de datos. Del total de alumnos encuestados, el $62.7 \%(n=79)$ tienen paquete de datos y el $37.3 \%(n=47)$ no lo tienen.

3. Asimismo, se consultó cuál es el uso que los alumnos daban prioritariamente al celular. Las respuestas con mayor frecuencia fueron las siguientes: (a) el 50\% ( $n=63)$ utilizan el celular para las redes sociales; (b) enviar y recibir mensajes $(21.4 \%, n=27)$; (c) utilizar multimedia $(18.3 \%, n=23)$.

4. Por último, se indagó la cantidad de tiempo que los alumnos utilizan el celular por día. Las respuestas fueron: (a) el 31\% ( $n=39)$ lo usa más de 4 horas; (b) el 27\% ( $n=34)$ lo usa entre dos y tres horas; (c) el 21.4\% ( $n=27)$ lo usa entre tres y cuatro horas; (d) el $13.5 \%(n=17)$ lo usa entre una y dos horas y (e) el 7.1\% $(n=9)$ lo usa menos de una hora por día.

\section{Discusión}

Se puede concluir que existe una correlación significativa entre la adicción al celular, medido por el TDM, y el rendimiento académico, lo cual valida nuestra hipótesis. Sin embargo, la correlación es débil, y esto puede entenderse debido a que el rendimiento académico está influenciado por una multiplicidad de factores (alimentación, socioeconómico, estado anímico, hábitos de estudio, etc.).

Asimismo, se encontró una correlación entre la actitud hacia la lectura y su repercusión académica en alumnos de educación secundaria. Esto muestra la importancia de formar a los estudiantes desde pequeños con una disposición favorable hacia la lectura, pues esto contribuirá a un mejor desempeño en los estudios.

Aunque las mujeres presentaron un mayor nivel de dependencia del celular, obtuvieron un promedio más alto que los varones en el rendimiento académico, medido 
por el promedio de calificaciones del primer trimestre de 2018. Estos resultados están en coherencia con un informe de la OCDE señala que las mujeres sacan mejores notas desde el colegio a la universidad y que tienen una menor tasa de fracaso escolar y de repitencia, donde se predice que el 66\% de las chicas irán a la universidad en los países de la OCDE, frente al 52\% de los varones. La nota media en España para mujeres fue de 7,04, frente a un promedio de 6,83 de los varones universitarios (Rivera, 2014). Asimismo, la prueba PISA (Programa Internacional para la Evaluación de Estudiantes), sobre una base de 18.000 escuelas de 74 países, mostró que las mujeres obtuvieron un mayor promedio de notas que los varones (Duque, 2015). Algunos explican esta diferencia al mencionar que, desde el punto de vista de la psicología, las mujeres tienden a madurar antes que los hombres; sin embargo, otros reconocen que esto ocurre y que es un tema poco explorado (El diario, 2014). Así, un estudio trató de identificar el peso de diferentes dimensiones del funcionamiento familiar sobre el rendimiento escolar por género en 630 estudiantes, y encontró que las mujeres tuvieron un mayor rendimiento escolar $(t=-3.053, p=.002)$ que los varones (Sarmiento Silva, Vargas Gallegos y Díaz Gutiérrez, 2012).

Por otro lado, una de las actividades que han declinado en la preferencia de uso del tiempo entre los jóvenes es la lectura. Esta es reconocida por ser el agente de aprendizaje primario; y es necesario promover desde el hogar y el aula el gusto por la lectura, ya que el factor emocional es una de las dimensiones que mayor impacto tuvo en la actitud hacia la lectura. El estudiante necesita esforzarse por lograr un aprendizaje profundo y ese esfuerzo implica dedicar tiempo a la lectura reflexiva. El éxito académico se forja diariamente. Las actitudes, los hábitos y la forma de administrar el tiempo, son llaves en manos de los estudiantes que deberían permitir el afianzamiento de motivaciones, estrategias y acciones adecuadas para gestionar su proyecto educativo de manera autorregulada, con miras a ser profesionales competentes (Hilt, 2013, p. 163).

\section{Referencias}

Aiken, L. R. (2003). Test psicológicos y evaluación. México: Pearson Educación.

Aliaga Jiménez, L. Y. (2012). Comprensión lectora y rendimiento académico en comunicación de alumnos del segundo grado de una institución educativa de Ventanilla. Tesis de Maestría, Universidad de San Ignacio de Loyola, Lima, Perú.

Bokhorst-Heng, W. y Pereyra, D. (2008). Non-at-risk adolescents' attitudes towards reading in a Singapore secondary school. Journal of Research in Reading, 31(3), 285-301. doi:10.1111/j.1467-9817.2008.00369.x 
Castelli Olvera, A. K. y Rosa, M. V. (2018). Del aula a las redes sociales: Cyberbullying en dos universidades de Pachuca, Hidalgo (México). Revista Científica de la Universidad Autónoma de Manizales, 25(44), 233-254.

Chóliz, M. y Villanueva, V. (2011). Evaluación de la adicción al móvil en la adolescencia. Revista Española de Drogodependencias, 36(2), 165-184.

Duque, G. (2015). Las mujeres son mejores estudiantes que los hombres. Recuperado de http://www.portafolio.co/internacional/mujeres-son-mejores-estudianteshombres-33630

Fishbein, M. y Ajzen, I. (1975). Belief, attitude, intention, and behavior: An introduction to theory and research. Reading, MA: Addison-Wesley.

Gamero, K., Flores, C., Arias, W. L., Ceballos, K. D, Román, A. y Marquina, E. (2016). Estandarización del Test de Dependencia al Celular para estudiantes universitarios de Arequipa. Persona, 19, 179-200.

Garbanzo Vargas, G. M. (2007). Factores asociados al rendimiento académico en estudiantes universitarios, una reflexión desde la calidad de la educación superior pública. Revista Educación, 31(1), 43-63.

García Martínez, V. y Fabila Echauri, A. M. (2014). Nomofobia vs. nomofibia, irrupción del teléfono móvil en las dimensiones de la vida de los jóvenes: un tema pendiente para los estudios en comunicación. Revista Razón y Palabra, 86, 682-696.

Guillén Cadena, D. M., Beltrán Lugo, N. I., Flores Atilano, B., Aristizábal Hoyos, G. P. y Garduño Magaña, A. (2016). Problemas de salud derivados del uso del teléfono celular. Revista Iberoamericana de Educación e Investigación en Enfermería, 6(4), 62-70.

González Cortijo, I. (2012). Estudio sobre el uso y abuso del teléfono móvil en alumnos de educación primaria. Recuperado del https://reunir.unir.net/bitstream/handle/123456789/992/2012_11_20_TFG_EST UDIO_DEL_TRABAJO.pdf?sequence=1\&isAllowed=y

Henríquez Ritchie, P., Organista Sandoval, J. y Lavigne, G. (2013). Nuevos procesos de interactividad e interacción social: uso de smartphones por estudiantes y docentes universitarios. Revista Electrónica Actualidades Investigativas en Educación, 13(3), 1-21.

Hilt, J. A. (2013). Adicción a Internet, enfoques de aprendizaje, hábitos y actitudes hacia la lectura, y su relación con la aptitud verbal y la aptitud matemática. Recuperado de http://dspace.biblioteca.um.edu.mx/jspui/handle/20.500.11972/ 147

Lainfiesta Stricker, M. E. (2006). Relación que existe entre la comprensión de lectura y el rendimiento académico de los alumnos de primero básico de Mixco Guatemala. Recuperado de http://biblioteca.usac.edu.gt/tesis/07/07_1757.pdf

Marcelino Bento, M. C. y dos Santos Cavalcante, R. (2013). Tecnologias Móveis em Educação: o uso do celular na sala de aula. ECCOM, 4(7), 113-120. 
McKenna, M. C., Kear, D. J. y Ellsworth, R. A. (1995). Children's attitudes toward reading: A national survey. Reading Research Quarterly, 30(4), 934-956.

Mendoza Méndez, R. V., Pérez Chávez, M. A., Jaramillo Jaramillo, M. y Baena Castro, G. R. (2018). Adicción al teléfono móvil y su relación con el rendimiento académico de los alumnos del Centro Universitario UAEM Temascaltepec. Revista Caribeña de Ciencias Sociales. Recuperado de www.eumed.net/ rev/caribe/2018/05/aiccion-telefono-alumnos.html

Morales Vallejo, P. (2011). Guía para construir cuestionarios y escalas de actitudes. Recuperado de http://www.upcomillas.es/personal/peter/otrosdocumentos/ Guiaparaconstruirescalasdeactitudes.pdf

Peiró i Gregòri, S., Merma Molina, G., Beresaluce Díez, R. y Moncho Pellicer, A. (2009). Carácter: formación de actitudes y hábitos. Recuperado de http://rua .ua.es/dspace/bitstream/10045/12416/3/15.\%20CARACTER\%20ACTITUDES $\% 20 \mathrm{Y} \% 20 \% 20 \mathrm{H} \% \mathrm{C} 3 \% 81 \mathrm{BITOS} . \mathrm{pdf}$

Ponce Aguirre, A. (2010). La actitud como factor determinante en el comportamiento. Recuperado de http://alexponceaguirre.suite101.net/la-actitud-como-factordeterminante-en-el-comportamiento-a18084

Rivera, R. (2014). Las chicas son mejores estudiantes y más exigentes, pero ellos destacan en mates. Recuperado de http://www.lainformacion.com/educacion/ las-chicas-son-mejores-estudiantes-y-mas-exigentes-pero-ellos-destacan-enmates_pAvYCVZCh2jxvxzmlsLtt/

Sarmiento Silva, C. Vargas Gallegos, M. y Díaz Gutiérrez, R. (2012). Diferencias en el rendimiento escolar por género asociadas al funcionamiento familiar de estudiantes de bachillerato. Quaderns de Psicología, 14(2), 17-23.

Tejada, A. (2008). ¿Tiene usted hábitos de lectura? Recuperado de http://consultorios .universia.edu.pe/2008/06/09/serie-animacion-a-la-lectura-desde-la-biblioteca/

UNESCO. (2015). La lectura en la era móvil: un estudio sobre la lectura móvil en los países en desarrollo. Recuperado de http://unesdoc.unesco.org/images/0023/002338/ 233828s.pdf 